\author{
論文 \\ Shock Tube Studies of the Development of Detached \\ Shock Waves in Transonic Flow-Part $I^{*}$ \\ Matsunosuke IWASAKI ${ }^{* *}$ and Kosuke UMETSU***
}

\begin{abstract}
Summary
The development of detached shock waves ahead of wedges of $10^{\circ}$ and $180^{\circ}$ total angle has been studied for Mach Numbers of 1.04 and 1.15. It has been found that (i) in the early state of development the process of development is considerably influenced by wedge angle, (ii) the dependency of detached shock wave-shape upon wedge angle is less pronounced as the flow Mach Number approaches one, and (iii) in the absence of shock tube wall effect geometrically similar models having the same value of $D / T_{0}$ (non-dimensional delay time) produce similar shapes of detached shock waves for the same Mach Number.
\end{abstract}

\section{Symbols}

$D=$ time after impact of incident shock front on leading edge of models

$\dot{U}=$ shock front velocity

$t \quad=$ thickness of wedges

$T_{0}=t / U$, time unit

$D / T_{0}=$ non-dimensional delayed time

$M \quad=$ Mach Number of warm air behind shock front

s $\quad=$ detached shock distance measured from shoulder of wedge

$x$ and $y=$ coordinates of points on detached shock wave

\section{Introduction}

The senior author has already reported some results on the development of detached shock waves $^{11}$. These were concerned, for the most part, with the process of development covering the whole range from the initial to the final stage. In this paper it is shown that by using models of different size, each step of the

* Received, April 8th, 1957.

* Assiatant Professor, Department of Applied Mechanics, Faculty of Engineering, Kyushu University.

** Shinmeiwa Industrial Company. Formerly postgraduate student of Department of Applied Mechanics, Faculty of Engineering, Kyughu University. development can be seen precisely. This method of experiment has given encouraging preliminary results and extended experiments are presently underway. The purpose of this paper is to present the results thus far obtained for the initial stage of detached shock wave development. The results of current and projected experiments will be given in a forthcoming paper.

2. Apparatus, Method of Experiment and Results Obtained

The shock tube used in the present work was the same as described in reference 1 with the dimensions of $59 \mathrm{~mm}$ (width), $150 \mathrm{~mm}$ (height) and $4,397 \mathrm{~mm}$ (length) and with the high pressure chamber maintained at one atmospheric pressure. The data reported were obtained at Mach Numbers of 1.04 and 1.15 with mild steel wedge models of $10^{\circ}$ and $180^{\circ}$ total angle and having thicknesses of $1,3,6,10$ and $20 \mathrm{~mm}$.

As shown in reference 1, if the formost points of detached shock waves ahead of geometrically similar models are plotted in the non-dimensional coordinates $s / t$ and $D / T_{0}$, the curve for each model follows a common envelope up to a certain value of $D / T_{0}$. This value of $D / T_{0}$ at which each curve departs from the common envelope 
varies with model thickness and test Mach Number as shown in Figure 2. When this critical value of $D / T_{0}$ is exceeded, the detached shock waves are distorted and their form obliterated before the contact surface reaches the test section.

The departure of each curve from the common curve seems to be due to the effect of the walls of the shock tube. Considering that the wall effect on the flow field about models of larger size should appear in the earlier stages of flow field development, it is found that the departure stated above occurs in accordance with this hypothesis. And if there are no wall effects, the $s / t$ curves obtained with various sizes of similar models should coincide with each other. The envelope of the individual $s / t$ curves, therefore, can be considered to be that true location of detached shocks which are not influenced by shock tube wall effect. Consequently, by graphically presenting data with $D / T_{0}$ as a corrdinate, the data not affected by wall effect can be distinguished from the data influenced by wall effects. Thus, by using the proper size of model for the stage of development under study, each step of the detached shock wave development can be obtained precisely. It is to be noted, however, that the wall effect is very strong for Mach Numbers very close to one and in the final stage of development of detached shocks in transonic flow. In these two cases, therefore, the strong wall effects preclude the application of the method described above. Now that $D / T_{0}$ is, as stated above, very useful in our

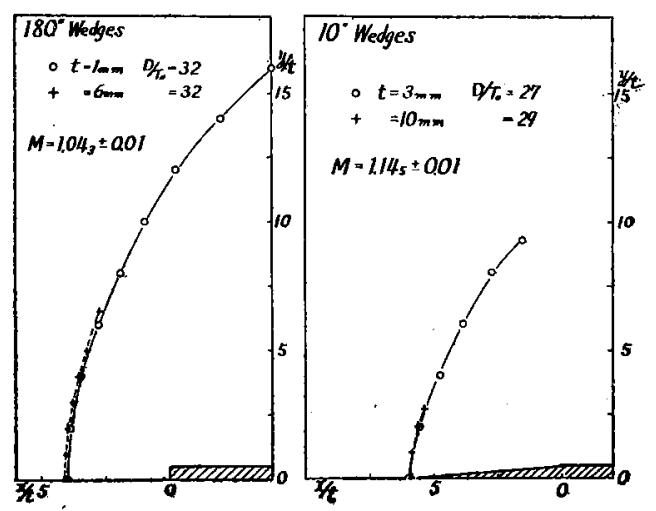

Fig. 1 Shape comparison of detached shocks analysis, the following data are shown as functions of $D / T_{0}$.

Figure 1 is the comparison of the shapes of detached shock waves. The left-hand figure corresponds to $180^{\circ}$ wedges for $M=1.04$ and $D / T_{0}$ $=32$, while the right-hand figure is for $10^{\circ}$ wedges at $M=1.15$ and $D / T_{0}=28$ (references 2 and 3 present the shapes of detached shock waves for eylinders with $M=1.05$ and $D / T_{0 \% 21)}$ The ratios of the model sizes are $6: 1$ and $3.33: 1$ respectively, so that models of considerably different sizes are compared. The agreement of the shapes which are drawn by non-dimensional coordinates is seen to be very good. Accordingly it may be said that the shapes of detached shocks. in the non-dimensional coordinated $x / y$ and $y / t$. are similar for geometrically similar models tested at the same values of Mach Number and non-dimensional delay time, $D / T_{0}$. This is true, of course, only when the wall effect of the shock tube is not present.

Figures 2 and 3 show the change of location. of the normal part of detached shock waves for. wedges of $10^{\circ}$ and $180^{\circ}$ respectively as the value. of $D / T_{0}$ increases. The thick lines give the correct values of $s / t$ without the effect of walls. These figures correspond especially to the initial stage of development while the curves in ref-
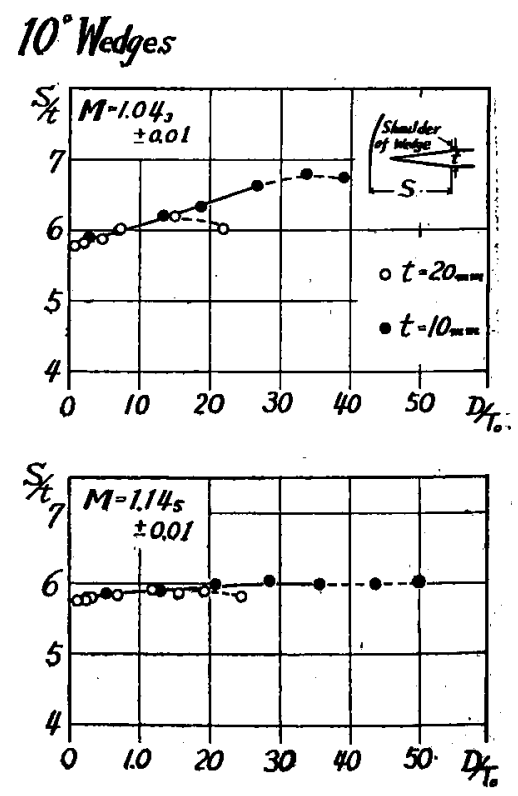

Fig. $2 s / t$ curve for wedge profile of $10^{\circ}$. 
$180^{\circ}$ Wedges
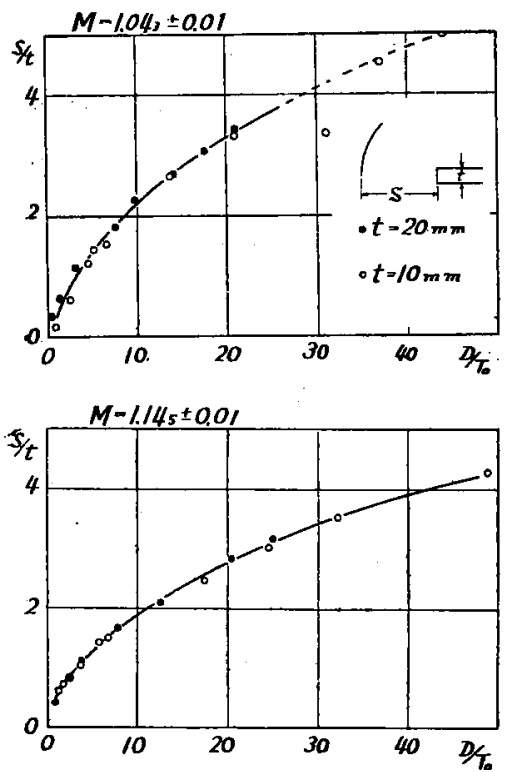

Fig. $3 \mathrm{~s} / t$ curve for wedge profile of $180^{\circ}$

erence 1 are for larger values of $D / T_{0}$. Comparing Figures 2 and 3 , it is seen that the $s / t$ curves for the same Mach Number differ from each other considerably. These two curves, however, coincide with each other when $D / T_{0}$ becomes equal to 100 for $M=1.04$ and 250 for $M=1.15$ (see Figure 11 of reference 1). In the initial stage of development the detached shock of the wedge of $10^{\circ}$ stays almost at the same position for the Mach Number of 1.15. When the Mach Number becomes 1.04, $s / t$ increases linearly, but slightly, with $D / T_{0}$ as seen in Figure 2. On the other hand, Figure 3 shows that, for the $180^{\circ}$ wedge, the value of $\mathrm{s} / t$ increases rapidly up to $D / T_{0}$ equal to 5 and thereafter the gradient of the $s / t$ curves gradually decreases.

The accompanying tabulation is convenient for

\begin{tabular}{|c|c|c|c|c|c|c|c|}
\hline \multicolumn{8}{|c|}{$M=1.04$} \\
\hline \multirow{2}{*}{ wedges } & \multirow[b]{2}{*}{$D / T_{0}$} & \multicolumn{3}{|c|}{$\mathrm{s} / t$} & \multicolumn{3}{|c|}{$s / t-(8 / t)_{0}$} \\
\hline & & 5 & 10 & 25 . & 5 & 10 & 25 \\
\hline $\begin{array}{c}10^{\prime} \\
180^{3}\end{array}$ & & $\begin{array}{l}5.9 \\
1.4\end{array}$ & $\begin{array}{l}6.1 \\
2.3\end{array}$ & $\begin{array}{l}6.55 \\
3.65\end{array}$ & $\begin{array}{l}0.18 \\
1.4\end{array}$ & $\begin{array}{l}0.38 \\
2.3\end{array}$ & $\begin{array}{l}0.83 \\
3.65\end{array}$ \\
\hline \multicolumn{8}{|c|}{$M=1.15$} \\
\hline $\begin{array}{r}10^{\circ} \\
180^{\circ}\end{array}$ & & $\begin{array}{l}5.8 \\
1.3\end{array}$ & $\begin{array}{l}5.9 \\
1.8\end{array}$ & $\begin{array}{l}6.0 \\
3.1\end{array}$ & $\begin{array}{l}0.08 \\
1.3\end{array}$ & $\begin{array}{l}0.18 \\
1.8\end{array}$ & $\begin{array}{l}0.28 \\
\mathbf{3 . 1}\end{array}$ \\
\hline
\end{tabular}

comparing the curves of Figures 2 and 3 at values of $D / T_{0}$ corresponding to 5,10 and 25 . In the table $(s / t)_{0}$ denotes the location of the detached shock at $D / T_{0}=0$, or the location of the leading edge of a wedge measured from the shoulder of the wedge, so that the values of $(s / t)_{0}$ are 5.72 and 0 for the $10^{\circ}$ and $180^{\circ}$ wedges respectively. The difference in the process of development of the detached waves ahead of the $10^{\circ}$ and $180^{\circ}$ wedges may be clearly seen from the table. The numbers in the right-hand column of the table show that the bow shock preceding the $180^{\circ}$ wedge advance into the oncoming flow much more rapidly than the detached shock ahead of the $10^{\circ}$ wedge.

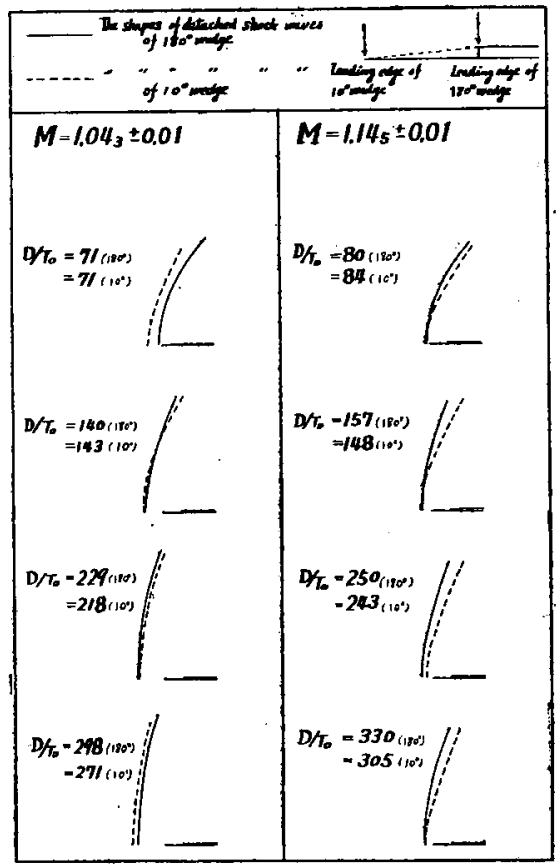

Fig. 4 Shape comparison in process of development

Finally in Figure 4 the shapes of detached bow shocks for different values of $D / T_{0}$ are compared in non-dimensional representation. As seen in the right-hand figure for $M=1.15$, the $10^{\circ}$ wedge bow shocks have different shapes from that of the $180^{\circ}$ wedge shocks for every value of $D / T_{0}$ even though the foremost points are the same. This difference, however, disappears when the Mach Number becomes 1.04, so that almost full 
coincjdence in shapes is obtained for values of $D / T_{0}$ larger than 200 as may be seen in the left-hand figure. From this fact we may say that, for Mach Numbers very close to one, the shape and location of detached shocks are independent of wedge angle in every stage of their development. Griffith' describes in his paper the independence of the shape of detached shock waves and wedge angle in the steady transonic flow. It is found by the work presented here that Griffith's result is true for the unsteady state too.

The effect of Reynolds Number on detached shock waves has not been considered in the present work. This effect, however, appears to be large for thinner models ${ }^{1)}$ and is to be investigated in future work.

\section{Conclusions}

From the results given above the following conclusions are drawn:

a. The early stages of development of detached shock waves are considerably influenced by wedge angle.

b. The shape of detached shock waves is less dependent on wedge angle as the flow Mach
Number approaches one.

c. If the values of $D / T_{0}$ at which the geometrically similar models are tested are the same and if the shock-tube wall effect is not. present in the flow field, the shapes of detached shock waves for the same Mach Number are the same.

These experiments were performed with theaid of the Collective Scientific Fund of theEducation Ministry. The thanks of the senior author are due to Mr. D. H. Daley for his kind. and careful correction of the manuscript throughout the preparation of it in English.

\section{References}

1) Iwasaki, M. : On the Errors of a Time Counting System for Ure in Fxperiments with a Shock Tube J. Japan Soc. Aero. Eng. Vol. 5, No. 40, (1957/5).

2) Iwasaki, M. : Shock-Tube Studies of the Development of Detached Shock Waves in the Transonic Flow, Pre-print of the Sixth Federated Lecture Meeting of Applied Mech. in Japan, No, 2, Hydro,. $(1956 / 10)$, p. 21, (in Japanege).

3) Iwasaki. M. : Studieg of the Development of De tached Shock Waves in the Transonic Region with a Shock Tube (I), Techno. Rep. Kyushu Univ., Vol. 29, No. 1, (1956/6), p. 22, (in Japanese).

4) Griffith, W.: Shock-Tube Studies of Transonic Flow over Wedge Profiles, J. Aero. Sci, Vol. 19m No. 4, (1952/4), p. 249.

\title{
啳退板翼の弾性特性についての実験と解析*

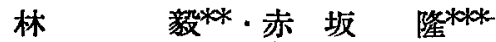

The Experiments and Analysis on the Elastic Properties of the Swept-Back Wing Plate

\section{Tsuyoshi HaYashi and Takashi AKasaka}

\begin{abstract}
As a basic investigation on the swept-back wing plate of the high speed aeroplanes, the experiments on the elastic properties of the $30^{\circ}$ swept back parallelogram wing plate with constant thickness in the two loading cases, i.e. (1) simple torsion, and (2). concentrated load were performed.

We analyzed the above two cases by $S-R-S$ method in which the deflection function $w(x, y)$ is assumed as follows,

$$
w=f(x)+g_{1}(x) \cdot y+g_{2}(x) \cdot y^{2},
$$

and assured the good coincidence between the experimental results and the theoretical ones.

It is interesting to note in particular, that our analytical stress distribution agree well with the experiments, even when the swept back angle is $30^{\circ}$, and that the invariant. axis, which may be called by the elastic axis refered to the aerodynamic chord, can be found when the aspect ratio of the wing is rather large.
\end{abstract}

